\title{
The assessment of circulating volume using inferior vena cava collapse index and carotid Doppler velocity time integral in healthy volunteers: a pilot study
}

\author{
Tom Peachey ${ }^{1}$, Andrew Tang ${ }^{1}$, Elinor C. Baker ${ }^{1}$, Jason Pott ${ }^{1}$, Yonathan Freund ${ }^{1,2}$ and Tim Harris ${ }^{1,3^{*}}$
}

\begin{abstract}
Background: Assessment of circulating volume and the requirement for fluid replacement are fundamental to resuscitation but remain largely empirical. Passive leg raise (PLR) may determine fluid responders while avoiding potential fluid overload. We hypothesised that inferior vena cava collapse index (IVCCI) and carotid artery blood flow would change predictably in response to PLR, potentially providing a non-invasive tool to assess circulating volume and identifying fluid responsive patients.
\end{abstract}

Methods: We conducted a prospective proof of concept pilot study on fasted healthy volunteers. One operator measured IVC diameter during quiet respiration and sniff, and carotid artery flow. Stroke volume (SV) was also measured using suprasternal Doppler. Our primary endpoint was change in IVCCI after PLR. We also studied changes in IVCCl after "sniff", and correlation between carotid artery flow and SV.

Results: Passive leg raise was associated with significant reduction in the mean inferior vena cava collapsibility index from 0.24 to $0.17(p<0.01)$. Mean stroke volume increased from 56.0 to $69.2 \mathrm{~mL}(p<0.01)$. There was no significant change in common carotid artery blood flow. Changes in physiology consequent upon passive leg raise normalised rapidly.

Discussion: Passive leg raise is associated with a decrease of IVCCI and increase in stroke volume. However, the wide range of values observed suggests that factors other than circulating volume predominate in determining the proportion of collapse with respiration.

Conclusion: In contrast to other studies, we did not find that carotid blood flow increased with passive leg raise. Rapid normalisation of post-PLR physiology may account for this.

Keywords: Inferior vena cava, Carotid Doppler, Passive leg raise, Circulating volume, Fluid resuscitation, Cardiac index

Abbreviations: Cl, Confidence interval; IQR, Interquartile range; IVC, Inferior vena cava; IVCCI, Inferior vena cava collapse index; PA, Pulmonary artery; PLR, Passive leg raise; SD, Standard deviation; SV, Stroke volume; VTI, Velocitytime integral

\footnotetext{
* Correspondence: tim.harris@bartshealth.nhs.uk

'Emergency Department, Royal London Hospital, Barts Health NHS Trust,

Whitechapel Rd, London E1 1BB, UK

${ }^{3}$ Emergency Department, Whipps Cross University Hospital, Barts Health NHS

Trust, London, UK

Full list of author information is available at the end of the article
} 


\section{Background}

Accurate assessment of circulating volume is challenging in the acute setting. Pulse and blood pressure are neither sensitive nor specific in identifying hypovolaemia $[1,2]$. Healthy adults may lose up to $30 \%$ of their circulating volume with little change in their vital signs [2]. Furthermore, clinical parameters may be abnormal for reasons other than changes in circulating blood volume, such as tachycardia secondary to pain, or altered physiology due to medication [3]. Resuscitation with intravenous fluid aims to increase cardiac output, improving tissue oxygenation. Defining the volume and rate of fluid administration remains largely empirical, despite its fundamental importance in patient care.

Passive straight leg raise (PLR) increases venous return and effective circulating volume. PLR non-invasively delivers a fluid challenge of around $300 \mathrm{~mL}$ to the central circulation, allowing assessment of fluid responsiveness without fluid administration [4, 5]. An increase in cardiac output of $>10-15 \%$ in response to PLR predicts fluid responsiveness and this technique has been validated in spontaneously breathing patients so may be of use to emergency physicians [6, 7].

The inferior vena cava (IVC) may be measured by ultrasound. The proportion that the IVC collapses with respiration is termed the inferior vena cava collapse index $\left(\mathrm{IVCCI}=\left(\mathrm{IVC}_{\max }-\mathrm{IVC}_{\min }\right) / \mathrm{IVC}_{\max }\right)$. This has been shown to indicate fluid status in children [8], ventilated patients $[9,10]$ and healthy volunteers $[11,12]$. Previous studies have demonstrated that IVCCI predicts fluid responsiveness in mechanically ventilated patients $[9,13]$ but data in spontaneously ventilating patients is less robust $[14,15]$. At the time of this study, no study has evaluated IVCCI in relation to PLR.

Oesophageal Doppler is widely used in the intensive care environment to assess cardiac output and fluid responsiveness $[16,17]$. Suprasternal Doppler measures blood flow through the aortic valve enabling noninvasive assessment of stroke volume and cardiac output (USCOM, USCOM Ltd., Sydney, Australia). Suprasternal Doppler has been validated against other measures of stroke volume in the emergency department, operating room and intensive care unit and shown to increase in response to PLR, [18, 19]. We therefore used this technique as a non-invasive reference standard for changes in stroke volume with a PLR in this study. Carotid arterial Doppler analysis is a simple, non-invasive method to assess circulatory flow. At the time of designing this study it had not been assessed as a surrogate measure of cardiac output or in response to PLR. Subsequently limited data has suggested it provides a good tool to assess fluid responsiveness $[4,20]$.

We hypothesised that IVCCI and carotid arterial flow would change predictably in response to a PLR, potentially providing non-invasive tools to assist clinicians in assessing the circulating volume and identifying fluid responsive patients. We tested this hypothesis by performing a pilot study on healthy volunteers after a period of no fluid intake.

\section{Methods}

\section{Setting and participants}

This prospective observational study was carried out in a non-clinical area within a UK Emergency Department. Ethical approval was granted by the National Research Ethics Service (NRES Ref: 13/LO/0472). Informed consent was obtained from all participants.

Healthy volunteers were recruited. The majority of participants were NHS students and medical or nursing staff. Inclusion criteria were: aged 18 years or over, able to straight leg raise and to comprehend written and spoken English. Exclusion criteria were pregnancy, body mass index $>40$, atrial fibrillation, known ventricular dysfunction, valvular heart disease, hypertension, diabetes mellitus, chronic lung disease, inability to lie flat comfortably or have legs raised to $45^{\circ}$. Participants were fasted from food and drink for $8 \mathrm{~h}$ prior to scanning to maximise the probability of fluid responsiveness and as many ED attendees have had minimal fluid intake in the hours prior to arrival.

\section{Measurements and endpoints}

Ultrasound measurements were carried out by one operator (TP) trained to College Emergency Medicine levelone standard. Additional vascular ultrasound training was obtained and measurements performed on 20 volunteers prior to commencing the study; images and technique were peer reviewed by an experienced operator $(\mathrm{TH})$. A single operator was used as this was a proof of concept pilot study and we wished to exclude inter-observer variation. Ultrasound images were recorded using a SonoSite Edge (SonoSite Inc., Bothell, Washington, USA). IVC measurements were made with a curvilinear $3.5-5 \mathrm{MHz}$ ultrasound transducer via the subcostal hepatic window just distal to the IVC-hepatic vein junction, approximately $3-5 \mathrm{~cm}$ distal to the right atrium, similar to previous studies [12]. The IVC was identified by phasic collapse with respiration, Doppler wave-form and compressibility (Fig. 1a). The right common carotid artery was identified by anatomy and waveform characteristics. Carotid blood flow measurements were with a linear $7 \mathrm{MHz}$ probe (Fig. 1b) [12].

Participants lay with legs flat and torso at $45^{\circ}$ for $5 \mathrm{~min}$. Pulse rate was recorded from pulse oximetry blood pressure was recorded with an automated oscillometric sphygmomanometer, and respiratory rate was recorded manually. Ultrasound measurements in longitudinal $B$ mode were made of the maximum and 

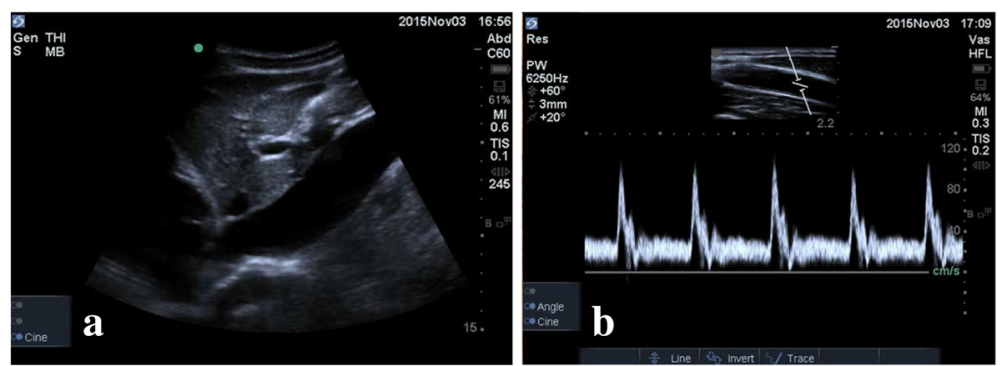

Fig. 1 Example IVC and carotid artery ultrasound images. a IVC diameter measurements were taken 3-5 cm distal to the IVC-right atrial junction. b Common carotid artery diameter and Doppler flow were measured $2 \mathrm{~cm}$ proximal to the carotid bulb

minimum diameter of the IVC occurring during 3 cycles of quiet respiration, and the smallest diameter occurring with a short sharp intake of breath ("sniff") [12]. IVCCI was calculated as: $\left(\mathrm{IVC}_{\max }-\mathrm{IVC}_{\min }\right) / \mathrm{IVC}_{\max }$. A sniff index was calculated $\left(\mathrm{IVC}_{\text {sniff }}\right)$ as: $\left(\mathrm{IVC}_{\max }-\mathrm{IVC}_{\text {sniff- }}\right.$ $\min ) / \mathrm{IVC}_{\max }$. Ultrasound cardiac output monitoring was used to measure the blood flow volume across the aortic valve to calculate stroke volume [18]. Longitudinal scans of the right common carotid artery were taken in B mode and Doppler used to calculate the minute flow volume (the total blood flow through the right common carotid artery over $1 \mathrm{~min}) 2 \mathrm{~cm}$ proximal to the carotid bulb using a $5 \mathrm{~mm}$ angle adjusted Doppler gate. The participant's torso was then lowered to horizontal and legs raised to 45 degrees. The measurements were taken immediately in the following order: suprasternal Doppler, IVCCI, then carotid flow and the participant returned to the initial position.

The primary endpoint was to assess the changes in IVCCI with usual respiration in response to PLR. Secondary endpoints were changes in the IVCCI with PLR using forced inspiration ('sniff'); changes in the carotid artery blood flow with PLR; and correlation between carotid blood flow with cardiac output and carotid velocity time integral (VTI) with stroke volume. We measured the changes in cardiac output with PLR for reference.

\section{Analysis}

All Gaussian distributed variables are expressed as mean and standard deviation (SD), and non-normally distributed variables were analysed as paired data and expressed as median and interquartile range (IQR). Means drawn from normally distributed data were compared with paired Student $\mathrm{T}$ test and non-parametric data were analysed with the paired Wilcoxon rank. Normality was assessed using Kolgomorov-Smirnov test. Correlation between stroke volume with carotid VTI and cardiac output with carotid flow was sought using Pearson test. A $p$-value of $<0.05$ was required for statistical significance, and all analyses were two-tailed. Data were analysed using SPSS (IBM SPSS Statistics 21.0, Chicago, Il, USA). No power calculation was possible for IVCCI and carotid Doppler changes with PLR as no previous work was available to base this upon, however, we estimated that a sample size of $>30$ subjects may be sufficient.

\section{Results}

Thirty-four participants were recruited into the study. Data for one participant was not recorded correctly and was excluded from analysis leaving 33 (17 male, 16 female) data sets. The physiological changes observed in this study are summarised in Table 1.

Table 1 Physiological changes with PLR

\begin{tabular}{|c|c|c|c|c|c|}
\hline Measurement/Indicator & Mean \pm (SD) legs flat & Mean $\pm(S D)$ legs raised & $\%$ change of mean with PLR & Mean difference & $p$ value \\
\hline Pulse rate & $61 \pm(10)$ & $60 \pm(9)$ & $-1.6 \%$ & $-1(95 \% \mathrm{Cl}-3:+1)$ & $>0.05$ \\
\hline Respiratory rate & $15 \pm(2)$ & $15 \pm(2)$ & $0.0 \%$ & $0(95 \% \mathrm{Cl}-1:+1)$ & $>0.05$ \\
\hline Systolic blood pressure & $115 \pm(11)$ & $107 \pm(10)$ & $-7.0 \%$ & $-8(95 \% \mathrm{Cl}-11:-5)$ & $>0.05$ \\
\hline Diastolic blood pressure & $72 \pm(8)$ & $65 \pm(8)$ & $-9.7 \%$ & $-7(95 \% \mathrm{Cl}-10:-4)$ & $>0.05$ \\
\hline IVC-CI - quiet respiration & $0.24 \pm(0.12)$ & $0.17 \pm(0.10)$ & $-29.2 \%$ & $-0.06(95 \% \mathrm{Cl}-0.10 ;-0.02)$ & $<0.01$ \\
\hline IVC-CI - sniff & $0.57 \pm(0.23)$ & $0.42 \pm(0.26)$ & $-26.3 \%$ & $-0.15(95 \% \mathrm{Cl}-0.25 ;-0.06)$ & $<0.01$ \\
\hline Stroke volume $(\mathrm{mL})$ & $56.0 \pm(13.0)$ & $69.2 \pm(14.2)$ & $+23.6 \%$ & $+13.2(95 \% \mathrm{Cl}+9.6:+16.7)$ & $<0.01$ \\
\hline Cardiac output (L/min) & $3.3 \pm(0.80)$ & $4.1 \pm(0.88)$ & $+22.3 \%$ & $+0.75(95 \% \mathrm{Cl}+0.55 ;+0.96)$ & $<0.001$ \\
\hline Common carotid flow (mL/min) & $770 \pm(202)$ & $773 \pm(167)$ & $+0.3 \%$ & $+3(95 \% \mathrm{Cl}-37:+43)$ & $>0.05$ \\
\hline
\end{tabular}

Changes in values of pulse rate, respiratory rate, blood pressure, cardiac output, IVCCl and carotid blood flow with PLR 
PLR was associated with significant reduction in mean inferior vena cava collapsibility index from 0.24 to 0.17 $(p<0.01)$ - mean difference of -0.06 (95\% CI -0.10 ; $-0.02)$. PLR was associated with a $23.6 \%$ increase in mean stroke volume from 56.0 to $69.2 \mathrm{~mL}(p<0.01)$, $18.2 \%$ increase in cardiac output from 3.3 to $4.1 \mathrm{~L} / \mathrm{min}$ $(p<0.001)$ - with a mean difference of $0.75 \mathrm{~L} / \mathrm{min}(95 \%$ CI $0.55 ; 0.96)$. Mean sniff collapsibility index reduced significantly from 0.57 to $0.42(p<0.01)$ - with a mean difference of -0.15 (95\% CI $-0.25 ;-0.06)$. There was no significant change in carotid blood flow, from $770 \mathrm{~mL} /$ $\min$ to $773 \mathrm{~mL} / \mathrm{min}(p=0.8)$.

Results of the IVC collapsibility index are summarised in Fig. 2a. The IVC collapsibility index decreases on passive leg raise. One participant fully collapsed their IVC with a sniff with legs supine or raised.

Figure $2 \mathrm{~b}$ shows PLR causes a significant rise in stroke volume from a median of $55 \mathrm{~mL}$ (IQR 49-63) to $69 \mathrm{~mL}$ (IQR 59-76). This demonstrates that stroke volume increases with passive leg raise as a consequence of increased central volume loading.

Figure 3 shows the correlation between IVCCI with legs supine, both with quiet respiration and sniff, and \% change in stroke volume with PLR. The data shows that although IVCCI reduces and stroke volume increases with passive leg raise (corresponding to the central fluid loading), there is poor correlation between IVCCI and stroke volume changes - IVC collapsibility does not predict the subjects in which fluid redistribution improves stroke volume.

Passive leg raise showed no significant alteration in carotid artery blood flow rate in our volunteers (Fig. 4).

The change in stroke volume on PLR was plotted against the change in carotid VTI with passive leg raise (Fig. 5). This shows poor correlation between changes in stroke volume with changes in common carotid flow. Poor correlation was also observed when absolute values of stroke volume and carotid VTI with legs supine $\left(r^{2}=0.21\right)$ or raised $\left(r^{2}=0.29\right)$ were compared.

\section{Discussion}

This study found that PLR was associated with a fall in IVCCI, increase in stroke volume and cardiac output, but no changes in carotid blood flow.

The magnitudes of IVCCI were greater with 'sniff' compared to quiet respiration, presumably due to greater changes in intrathoracic pressure. Mean reduction of IVCCI with PLR was similar with quiet respiration $(29.2 \%)$ and sniff $(26.3 \%)$ as a proportion of initial IVCCI.

We have shown that IVCCI, both with quiet respiration and 'sniff', decreases with PLR, suggesting that IVCCI alters with centralised blood volume and so may have potential to predict fluid responsiveness in spontaneously ventilating patients. However, we also found that the correlation between IVCCI and cardiac output was poor. The effect of PLR on IVCCI showed wide variation: ranging from a $96.6 \%$ reduction in quiet respiration IVCCI with PLR and a $92.4 \%$ reduction in sniff IVCCI with PLR to a decrease in IVCCI on PLR: opposite to the expected finding. Eight participants had a greater IVCCI post-PLR with quiet inspiration and five post-PLR with 'sniff' inspiration. These data suggest that variations in inspiratory force and volume may have a greater effect on IVCCI (and cardiac output) than volume centralisation with PLR in some subjects. The position change may affect respiratory effort and mechanics. Variability of IVCCI with breathing method has been reported by Kimura, who noted for the same inspiratory tidal volume diaphragmatic breathing caused greater IVCCI than chest wall breathing [21]. Thus IVCCI may have limited clinical potential unless inspiratory effort is standardised both between measurements and participants. Future work exploring the role of IVCCI should attempt to standardise respiratory effort.

Since our study was performed others have also explored the role of IVCCI and PLR. Corl [22] examined changes in IVCCI with PLR in spontaneously ventilating patients presenting to an adult emergency department, finding a mean IVCCI of $15.8 \%$, with IVCCI 4 min after PLR decreasing by an average of $0.5 \%$. The authors
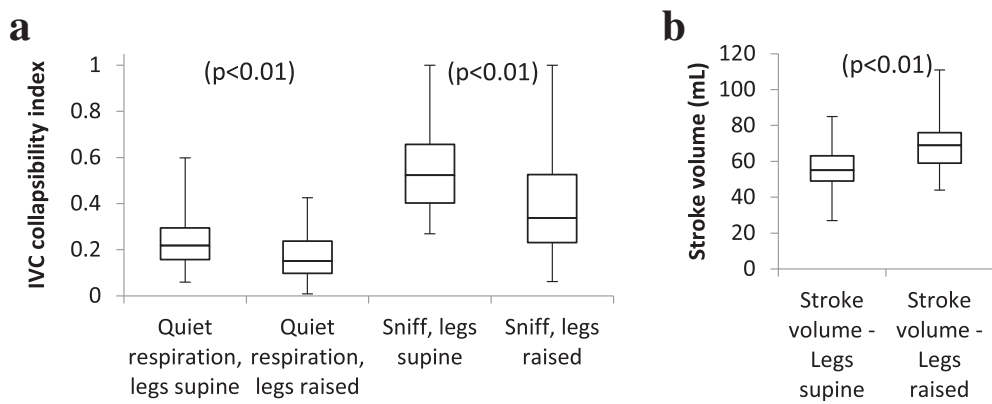

Fig. 2 Respiratory changes of IVC collapsibility and changes of stroke volume on passive leg raise. (a) Box plot illustrating change in IVC collapsibility index for quiet respiration and sniff on passive leg raise. (b) Box plot illustrating change in stroke volume on passive leg raise 


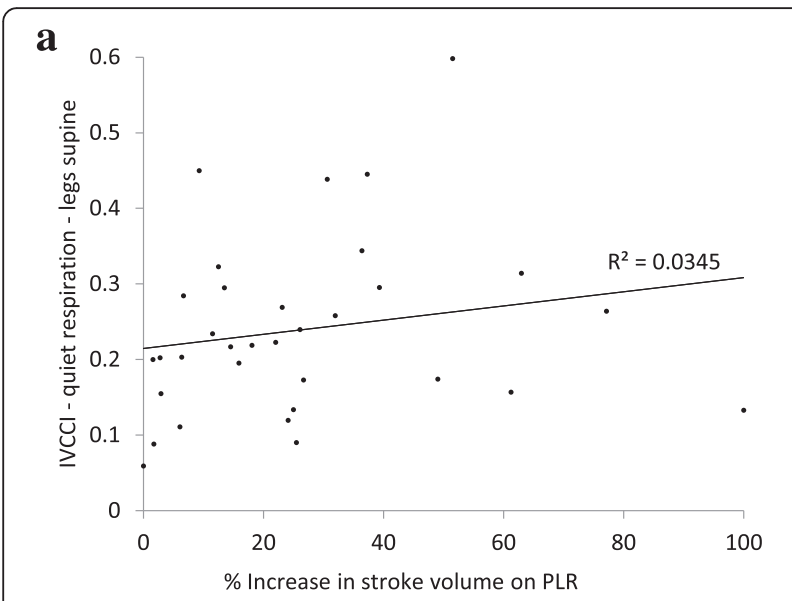

b

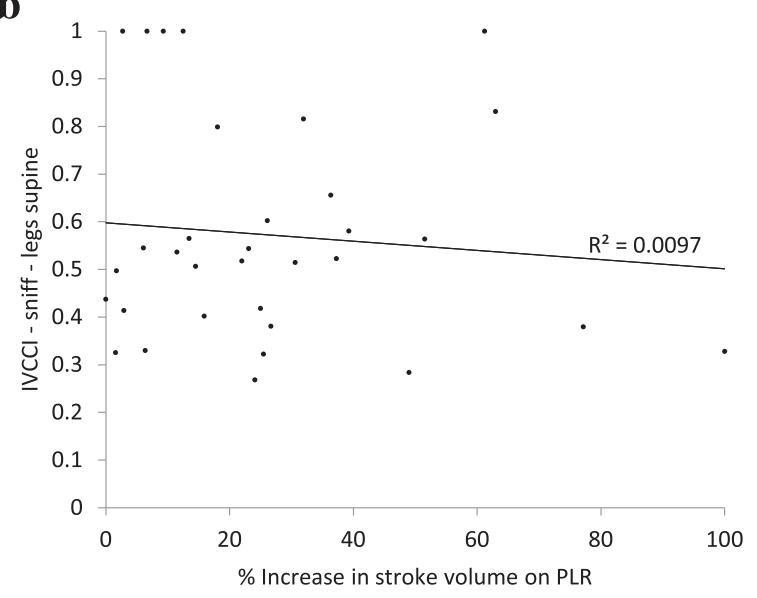

Fig. 3 Correlations between IVCCI with the change in stroke volume on passive leg raise. (a) Correlation between IVCCI with quiet respiration and increase in stroke volume on passive leg raise. (b) Correlation between IVCCI with a sniff and increase in stroke volume on passive leg raise

concluded IVCCI was a poor predictor of fluid responsiveness. This may also be consequent upon the 4-min delay between PLR and IVCCI measurement, as haemodynamic changes following PLR are transient as discussed further below. Panebianco [23] showed that IVC

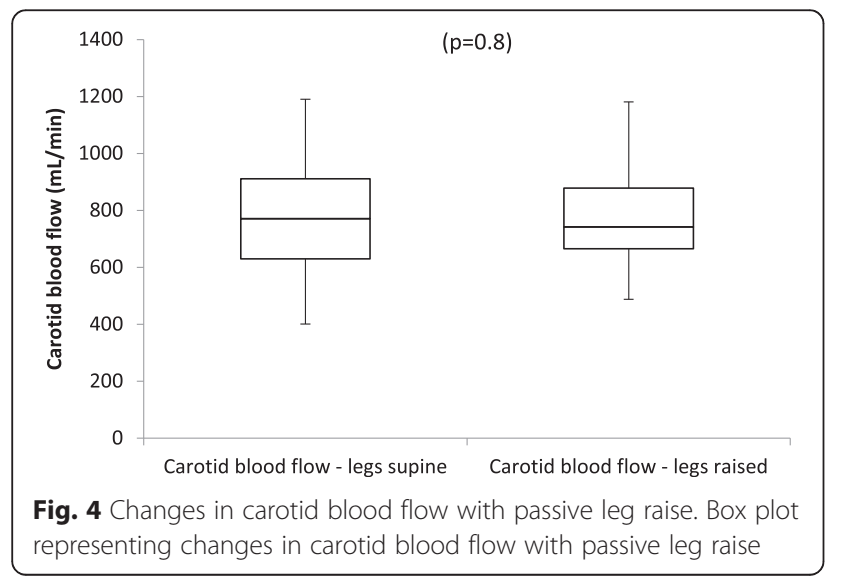

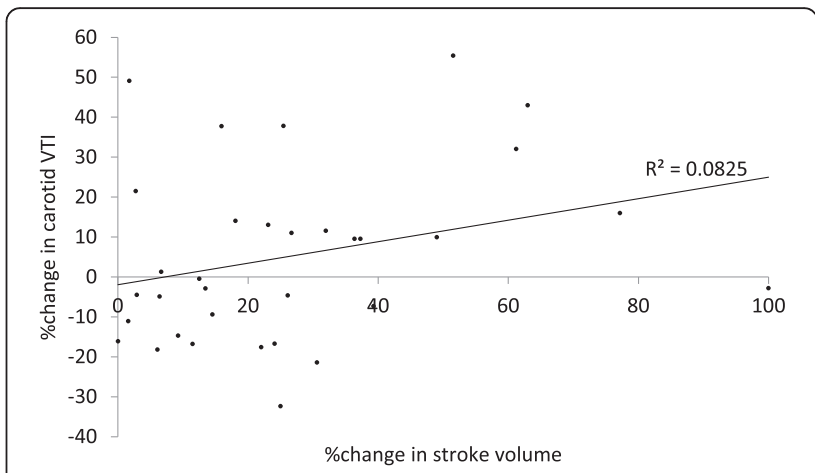

Fig. 5 Correlation between change in carotid VTI with PLR and increase in stroke volume on passive leg raise

size and IVCCI are little changed when recorded supine or at 45 degrees, concluding that measurements taken supine and semi-upright may be regarded as equivalent. Lanspa examined IVCCI in 14 spontaneously ventilating ICU patients receiving $10 \mathrm{~mL} / \mathrm{kg}$ intravenous fluid delivered over 20 min [24]. Fluid responsiveness was defined as increase in cardiac output of $>15 \%$. Five subjects were fluid responders and nine were non-responders. An IVCCI of $>15 \%$ had a positive predictive value of $62 \%$, but excellent negative predictive value of $100 \%(p=0.03)$. Muller examined 40 spontaneously ventilating ICU patients with acute circulatory failure [25]. Muller found that IVCCI of $>40 \%$ had a positive predictive value of $72 \%$ for fluid responsiveness (defined by increase in subaortic VTI of $>15 \%$ following infusion of $500 \mathrm{~mL} 6 \%$ hydroxyethylstarch), but IVCCI of $<40 \%$ had a negative predictive value of $83 \%$ and so could not exclude fluid responsiveness. Airapetian examined 59 spontaneously breathing ICU patients with acute circulatory failure [26]. This study found that an IVCCI of $>42 \%$ distinguished between fluid responders ( $>10 \%$ increase in cardiac output with $500 \mathrm{~mL}$ $0.9 \%$ saline infusion) and non-responders with high specificity ( $97 \%$ ) and positive predictive value (90 \%) but low sensitivity.

In our study the changes in cardiac output measured by USCOM were short-lived and values returned to their pre-PLR values within around $30 \mathrm{~s}$ to $2 \mathrm{~min}$. A recent study concurs; reporting that PLR results in a transient change in physiological measurements, with normal physiology restored within one to $4 \mathrm{~min}$ [27]. The time taken to restore to normal physiology was not an a priori measured variable in our study, as this rapid normalisation of physiology was not anticipated. However, this was noted consistently in all subjects, anecdotally, more rapidly in fitter and more athletic subjects.

The time to maximum change in stroke volume and return to baseline with PLR is not well defined. A study of healthy spontaneously ventilating volunteers by Delerme 
[6], measured changes in pulse oximetry waveforms with PLR, finding a significant difference between baseline and post-PLR, which were maintained for $5 \mathrm{~min}$. Boulain reported haemodynamic changes persisting for $4 \mathrm{~min}$ post-PLR in mechanically ventilated ICU patients [28]. However, Lamia [27] notes that haemodynamic changes following PLR are transient so designed their study to record their data within $1 \mathrm{~min}$ of PLR. Best practice for performing a passive leg raise is discussed by Monnet [29], who notes that physiological effects from PLR may vanish after $1 \mathrm{~min}$. This paper outlines how PLR should be optimally performed to assess for changes in stroke volume and discusses how differences in results in the literature may be due to differences in methods of PLR. The short duration of the PLR associated changes in stroke volume were not known at the time of conducting this study and consequently the exact times of each component of the study were not recorded. This may explain the range of changes in cardiac output reported here. The limited time window during which measurements should be taken potentially limits the utility of PLR without continuous cardiac monitoring.

Mean stroke volume increased $23.6 \%$ from 56.0 to $69.2 \mathrm{~mL}$. Mean cardiac output increased by $22.3 \%$ from $3.3 \mathrm{~L} / \mathrm{min}$ to $4.1 \mathrm{~L} / \mathrm{min}$ with PLR, comparable to other studies [6, 26, 27]. A rise in cardiac output $>10 \%$ with $500 \mathrm{~mL}$ crystalloid bolus or PLR is commonly used to define fluid responsive patients $[9,10]$. In our study 24 participants increased their cardiac output by $>10 \%$ on PLR and were therefore fluid responsive. Nine participants had changes in cardiac output of $<10 \%$. This is surprising as all participants were expected fluid responsive as they had normal cardiovascular systems, were well and fasted for $8 \mathrm{~h}$. Review of the quality of the suprasternal Doppler traces identified them as comparable to previous work and do not account for the nonresponders. This finding may reflect the transient effect on cardiac output consequent upon PLR. The USCOM cardiac output readings in this study are lower than may be expected for healthy volunteers. A related study on this machine performed by a different operator within our institution also obtained lower than expected cardiac index readings [12]. A meta-analysis by Chong and Peyton [30] comparing USCOM with thermodilution noted that USCOM underestimates cardiac output by an average of $0.39 \mathrm{~L} / \mathrm{min}$ with large variation between these methods of cardiac output measurement.

Our study reports that common carotid artery flow does not increase in response to PLR and we found no relationship between carotid artery blood flow and stroke volume, irrespective of leg positioning. This suggests that carotid VTI cannot be used as a surrogate measure for changes in stroke volume on PLR in healthy volunteers. This is contrary to a recent study by Marik where carotid artery blood flow differentiated fluid responders from non-responders [4]. In their intensive care unit based study of 34 patients, 17 non-responders to fluid boluses had minimal changes (mean increase $0.1 \%$ ) to carotid blood flow on PLR, while 17 fluid responders had a mean carotid blood flow increase of $79 \%$. This paper notes that work in their group on healthy volunteers found PLR caused an average $33 \%$ increase in stroke volume and $16 \%$ increase in carotid blood flow. However, there are differences in study design.

The Marik study measured the diameter of the carotid artery and found that this changed with fluid loading. Our study did not measure the carotid artery diameter, assuming no change with PLR, and thus our results may represent a false negative finding. Marik studied patients with septic shock in intensive care who had been partially or completely fluid resuscitated. Carotid blood flow changes in response to PLR maybe different in patients with sepsis as compared to healthy volunteers, possibly as a consequence of altered autoregulation [31]. As discussed, the changes in stroke volume consequent upon PLR are short lived and the carotid artery measurements were made at the end of our protocol, so delay in obtaining readings may result in recordings already returning to baseline. Gassner [20] also investigated the role of carotid artery ultrasound as a surrogate marker for cardiac output Their study on ICU patients found good correlation between carotid artery flow when compared with PA catheter (intra-class correlation 0.74) or arterial waveform pulse contour analysis (intra-class correlation 0.84 ) over a wide range of cardiac outputs.

\section{Limitations}

There are several limitations to this study. The study measurements were all taken by one operator (TP). Our data consequently allows no comment on inter-observer reliability. However, this was a pilot study designed to test our hypothesis in healthy volunteers and not the clinical utility of IVCCI or carotid blood flow with PLR. Secondly, this pilot proof of concept study was performed using healthy volunteers and not patients. Therefore, the results are not transferable to the clinical environment. We aimed to use this work to inform future clinical studies.

IVCCI is dependent on factors other than circulating volume. The rate and volume of inspiration, pulmonary pathology and cardiac disease all impact the intrathoracic pressure. Studies on mechanically ventilated patients standardise tidal volume by ideal body weight $[9,10]$. We believe that future studies exploring the role of IVCCI should focus on controlling and standardising these parameters if IVCCI is to find a role in spontaneously ventilating patients. 
In our study we found that changes in physiology following PLR were transient and rapidly normalise, often returning to baseline values in around $1 \mathrm{~min}$ following PLR. Obtaining readings for IVCCI, carotid blood flow and cardiac output within this time frame is challenging using the non-invasive technology evaluated in this study. The time required all data acquisition was not formally recorded and it is likely that some values were obtained after this time period, while the physiological responses to PLR were diminishing.

\section{Conclusions}

This study reports an increase in stroke volume and fall in IVCCI with PLR in healthy volunteers. The wide range of IVCCIs reported with PLR suggests that the technique requires development prior to clinical use. In contrast to previous studies we report that common carotid artery blood flow does not change with PLR. The haemodynamic changes on the healthy volunteers following PLR were transient and rapidly returned to the baseline values, which may account for these findings. This may limit the role of PLR as an indicator of fluid responsiveness when using non-invasive monitors unable to provide continuous readings.

\section{Acknowledgements}

We thank Benjamin Bussman for assistance in producing the example ultrasound images used in Fig. 1.

\section{Funding}

TP received a grant from the Isaac Schapera Trust for medical research. The funding body had no influence in the design of the study or the collection, analysis and interpretation of data or in the writing of the manuscript.

\section{Availability of data and material}

The datasets generated and analysed during the current study available from the corresponding author on reasonable request.

\section{Authors' contributions}

TP ran the study and collected data, TH, reviewed images collected. TP, TH, YF and AT provided manuscript formation. TP, ECB, JP, TH provided study design, training and supervision, TP, AT, YF and TH provided data analysis and interpretation. All authors read and approved the final manuscript.

\section{Authors' information}

Not applicable.

\section{Competing interests}

The authors declare that they have no competing interests.

\section{Consent for publication}

Written informed consent for the publication from the participants enrolled in this study may be provided on request.

\section{Ethics approval and consent to participate}

Ethical approval was obtained from the National Research Ethics Service (REC ref; 13/LO/0472) and the local ethics committee. All participants were informed of the purpose of the study and gave full written consent.

\section{Author details}

'Emergency Department, Royal London Hospital, Barts Health NHS Trust, Whitechapel Rd, London E1 1BB, UK. ${ }^{2}$ Sorbonne université, UPMC univ Paris-06, Paris, France. ${ }^{3}$ Emergency Department, Whipps Cross University Hospital, Barts Health NHS Trust, London, UK.
Received: 29 March 2016 Accepted: 22 August 2016

Published online: 02 September 2016

\section{References}

1. Dabrowski GP, Steinberg SM, Ferrara JJ, Flint LM. A critical assessment of endpoints of shock resuscitation. Surg Clin North Am. 2000;80:825-44.

2. Wo CC, Shoemaker WC, Appel PL, Bishop MH, Kram HB, Hardin E. Unreliability of blood pressure and heart rate to evaluate cardiac output in emergency resuscitation and critical illness. Crit Care Med. 1993;21:218-23.

3. Longmore JM, editor. Cardiovascular drugs. Oxf. Handb. Clin. Med. 8th ed. Oxford; New York: Oxford University Press; 2010. p. 108.

4. Marik PE, Levitov A, Young A, Andrews L. The use of bioreactance and carotid Doppler to determine volume responsiveness and blood flow redistribution following passive leg raising in hemodynamically unstable patients. Chest. 2013;143:364-70.

5. Maizel J, Airapetian N, Lorne E, Tribouilloy C, Massy Z, Slama M. Diagnosis of central hypovolemia by using passive leg raising. Intensive Care Med. 2007; 33:1133-8.

6. Delerme S, Renault R, Le Manach Y, Lvovschi V, Bendahou M, Riou B, et al. Variations in pulse oximetry plethysmographic waveform amplitude induced by passive leg raising in spontaneously breathing volunteers. Am J Emerg Med. 2007;25:637-42

7. Delerme S, Castro S, Freund Y, Nazeyrollas P, Josse M-O, Madonna-Py B, et al. Relation between pulse oximetry plethysmographic waveform amplitude induced by passive leg raising and cardiac index in spontaneously breathing subjects. Am J Emerg Med. 2010:28:505-10.

8. Levine AC, Shah SP, Umulisa I, Munyaneza RBM, Dushimiyimana JM, Stegmann K, et al. Ultrasound assessment of severe dehydration in children with diarrhea and vomiting. Acad Emerg Med Off J Soc Acad Emerg Med. 2010:17:1035-41.

9. Barbier C, Loubières Y, Schmit C, Hayon J, Ricôme J-L, Jardin F, et al. Respiratory changes in inferior vena cava diameter are helpful in predicting fluid responsiveness in ventilated septic patients. Intensive Care Med. 2004; 30:1740-6.

10. Feissel M, Michard F, Faller J-P, Teboul J-L. The respiratory variation in inferior vena cava diameter as a guide to fluid therapy. Intensive Care Med. 2004:30:1834-7.

11. Lyon M, Blaivas M, Brannam L. Sonographic measurement of the inferior vena cava as a marker of blood loss. Am J Emerg Med. 2005;23:45-50.

12. Baker EC, Pott J, Khan F, Freund Y, Harris T. Best approach to measuring the inferior vena cava in spontaneously ventilating patients: a pilot study. Eur J Emerg Med Off J Eur Soc Emerg Med. 2015;22:58-61.

13. Vieillard-Baron A, Chergui K, Rabiller A, Peyrouset O, Page B, Beauchet A, et al. Superior vena caval collapsibility as a gauge of volume status in ventilated septic patients. Intensive Care Med. 2004;30:1734-9.

14. Weekes AJ, Tassone HM, Babcock A, Quirke DP, Norton HJ, Jayarama K, et al. Comparison of serial qualitative and quantitative assessments of caval index and left ventricular systolic function during early fluid resuscitation of hypotensive emergency department patients. Acad Emerg Med Off J Soc Acad Emerg Med. 2011;18:912-21.

15. Yanagawa Y, Sakamoto T, Okada Y. Hypovolemic shock evaluated by sonographic measurement of the inferior vena cava during resuscitation in trauma patients. J Trauma. 2007;63:1245-8.

16. Valtier B, Cholley BP, Belot JP, de la Coussaye JE, Mateo J, Payen DM. Noninvasive monitoring of cardiac output in critically ill patients using transesophageal Doppler. Am J Respir Crit Care Med. 1998;158:77-83.

17. Schmid ER, Spahn DR, Tornic M. Reliability of a new generation transesophageal Doppler device for cardiac output monitoring. Anesth Analg. 1993;77:971-9.

18. van Lelyveld-Haas LEM, van Zanten ARH, Borm GF, Tjan DHT. Clinical validation of the non-invasive cardiac output monitor USCOM-1A in critically ill patients. Eur J Anaesthesiol. 2008;25:917-24.

19. Thiel SW, Kollef MH, Isakow W. Non-invasive stroke volume measurement and passive leg raising predict volume responsiveness in medical ICU patients: an observational cohort study. Crit Care Lond Engl. 2009;13:R111.

20. Gassner M, Killu K, Bauman Z, Coba V, Rosso K, Blyden D. Feasibility of common carotid artery point of care ultrasound in cardiac output measurements compared to invasive methods. J Ultrasound. 2014;18:127-33.

21. Kimura BJ, Dalugdugan R, Gilcrease GW, Phan JN, Showalter BK, Wolfson T. The effect of breathing manner on inferior vena caval diameter. Eur. J. Echocardiogr. J. Work. Group Echocardiogr. Eur. Soc. Cardiol. 2011;12:120-3. 
22. Corl K, Napoli AM, Gardiner F. Bedside sonographic measurement of the inferior vena cava caval index is a poor predictor of fluid responsiveness in emergency department patients. Emerg Med Australas EMA. 2012;24:534-9.

23. Panebianco NL, Shofer F, Cheng A, Fischer J, Cody K, Dean AJ. The effect of supine versus upright patient positioning on inferior vena cava metrics. Am J Emerg Med. 2014;32:1326-9.

24. Lanspa MJ, Grissom CK, Hirshberg EL, Jones JP, Brown SM. Applying dynamic parameters to predict hemodynamic response to volume expansion in spontaneously breathing patients with septic shock. Shock Augusta Ga. 2013;39:155-60.

25. Muller L, Bobbia X, Toumi M, Louart G, Molinari N, Ragonnet B, et al. Respiratory variations of inferior vena cava diameter to predict fluid responsiveness in spontaneously breathing patients with acute circulatory failure: need for a cautious use. Crit Care. 2012;16:R188.

26. Airapetian N, Maizel J, Alyamani O, Mahjoub Y, Lorne E, Levrard M, et al. Does inferior vena cava respiratory variability predict fluid responsiveness in spontaneously breathing patients? Crit. Care [Internet]. 2015 [cited 2016 Jul 30] 19. Available from: http://www.ncbi.nlm.nih.gov/pmc/articles/PMC4643539/.

27. Lamia B, Ochagavia A, Monnet X, Chemla D, Richard C, Teboul J-L. Echocardiographic prediction of volume responsiveness in critically ill patients with spontaneously breathing activity. Intensive Care Med. 2007;33:1125-32.

28. Boulain T, Achard J-M, Teboul J-L, Richard C, Perrotin D, Ginies G. Changes in BP induced by passive leg raising predict response to fluid loading in critically ill patients. Chest. 2002;121:1245-52.

29. Monnet $X$, Teboul J-L. Passive leg raising: five rules, not a drop of fluid! Crit. Care [Internet]. 2015 [cited 2016 Aug 8];19. Available from: http://www.ncbi. nlm.nih.gov/pmc/articles/PMC4293822/.

30. Chong SW, Peyton PJ. A meta-analysis of the accuracy and precision of the ultrasonic cardiac output monitor (USCOM). Anaesthesia. 2012;67:1266-71.

31. Hall JE. Guyton Hall Textb. Med. Physiol. 12th ed. Philadelphia: Saunders/ Elsevier; 2011. p. 761-70

\section{Submit your next manuscript to BioMed Central and we will help you at every step:}

- We accept pre-submission inquiries

- Our selector tool helps you to find the most relevant journal

- We provide round the clock customer support

- Convenient online submission

- Thorough peer review

- Inclusion in PubMed and all major indexing services

- Maximum visibility for your research

Submit your manuscript at www.biomedcentral.com/submit
Biomed Central 